\title{
Circulating osteocrin stimulates bone growth by limiting C-type natriuretic peptide clearance
}

\author{
Yugo Kanai, ${ }^{1}$ Akihiro Yasoda, ${ }^{1}$ Keita P. Mori, ${ }^{2}$ Haruko Watanabe-Takano, ${ }^{3}$ Chiaki Nagai-Okatani, ${ }^{4}$ Yui Yamashita, ${ }^{1}$ Keisho Hirota, ${ }^{1}$ \\ Yohei Ueda, ${ }^{1}$ Ichiro Yamauchi, ${ }^{1}$ Eri Kondo, ${ }^{1}$ Shigeki Yamanaka, ${ }^{5}$ Yoriko Sakane, ${ }^{1}$ Kazumasa Nakao, ${ }^{5}$ Toshihito Fujii, ${ }^{1}$ \\ Hideki Yokoi, ${ }^{2,6}$ Naoto Minamino, ${ }^{4}$ Masashi Mukoyama, ${ }^{6,7}$ Naoki Mochizuki, ${ }^{3,6}$ and Nobuya Inagaki ${ }^{1}$ \\ 1Department of Diabetes, Endocrinology and Nutrition, and ${ }^{2}$ Department of Nephrology, Kyoto University Graduate School of Medicine, Kyoto, Japan. ${ }^{3}$ Department of Cell Biology and ${ }^{4}$ Department of \\ Molecular Pharmacology, National Cerebral and Cardiovascular Center Research Institute, Osaka, Japan. ${ }^{5}$ Department of Oral and Maxillofacial Surgery, Kyoto University Graduate School of Medicine, Kyoto, \\ Japan. ' Japan Agency for Medical Research and Development-Core Research for Evolutionary Medical Science and Technology (AMED-CREST), National Cerebral and Cardiovascular Center Research Institute, \\ Osaka, Japan. ${ }^{7}$ Department of Nephrology, Kumamoto University Graduate School of Medical Sciences, Kumamoto, Japan.
}

\begin{abstract}
Although peptides are safe and useful as therapeutics, they are often easily degraded or metabolized. Dampening the clearance system for peptide ligands is a promising strategy for increasing the efficacy of peptide therapies. Natriuretic peptide receptor B (NPR-B) and its naturally occurring ligand, C-type natriuretic peptide (CNP), are potent stimulators of endochondral bone growth, and activating the CNP/NPR-B system is expected to be a powerful strategy for treating impaired skeletal growth. CNP is cleared by natriuretic peptide clearance receptor (NPR-C); therefore, we investigated the effect of reducing the rate of CNP clearance on skeletal growth by limiting the interaction between CNP and NPR-C. Specifically, we generated transgenic mice with increased circulating levels of osteocrin (OSTN) protein, a natural NPR-C ligand without natriuretic activity, and observed a dose-dependent skeletal overgrowth phenotype in these animals. Skeletal overgrowth in OSTN-transgenic mice was diminished in either CNP- or NPR-C-depleted backgrounds, confirming that CNP and NPR-C are indispensable for the bone growth-stimulating effect of OSTN. Interestingly, double-transgenic mice of CNP and OSTN had even higher levels of circulating CNP and additional increases in bone length, as compared with mice with elevated CNP alone. Together, these results support OSTN administration as an adjuvant agent for CNP therapy and provide a potential therapeutic approach for diseases with impaired skeletal growth.
\end{abstract}

\section{Introduction}

Most bones in mammals are formed through endochondral ossification, in that cartilaginous anlage is formed first and is subsequently replaced by calcified bone tissue (1). Although many signaling pathways, including Indian hedgehog/parathyroid hormone-related protein (PTHrP), fibroblast growth factor, b one morphogenetic protein, Sry (sex determining region Y)-box 9 (SOX9), and runt-related transcription factor 2 (RUNX2) signaling, have been shown to be engaged in the process of endochondral ossification (2), few factors are known to stimulate bone growth. C-type natriuretic peptide (CNP), a member of the natriuretic peptide family, is a rare factor that has the potential to stimulate endochondral ossification and elongate bones, as evidenced by the skeletal phenotypes of transgenic and knockout mice (3-5). So far, 2 receptors for CNP have been identified (6). One is a subtype of membranous guanylyl cyclase, natriuretic peptide receptor $\mathrm{B}$ (NPR-B), which is a biologically active receptor that mediates signaling by producing intracellular cGMP, a second messenger. In fact, NPR-B and CNP are both expressed in the cartilaginous growth plate, and homozygous loss-of-function

Conflict of interest: The authors have declared that no conflict of interest exists. Submitted: May 9, 2017; Accepted: August 29, 2017.

Reference information: / Clin Invest. 2017;127(11):4136-4147.

https://doi.org/10.1172/JCI94912. mutations of NPR-B not only in murine models $(7,8)$ but also in the human disorder acromesomelic dysplasia type Maroteaux $(9,10)$ cause impaired skeletal growth. In addition, heterozygous loss-offunction mutations of NPR-B have been reported to cause short stature and mild skeletal defects (11-15), and recently heterozygous loss-of-function mutations of CNP have also been shown to cause similar skeletal defects to those of NPR-B (16). Furthermore, a recent case series showed that gain-of-function mutations in NPR-B cause a skeletal overgrowth phenotype $(17,18)$, demonstrating the ability of CNP/NPR-B signaling to stimulate bone growth in humans. On the other hand, natriuretic peptide clearance receptor (NPR-C), which lacks the intracellular domain of the receptor, is thought to be engaged in clearing natriuretic peptide ligands.

In humans, impaired skeletal growth is caused by disturbance of endochondral ossification and occurs in various conditions, including skeletal dysplasia, a large and heterogeneous group of inherited skeletal disorders (19). Based on the specific effects of CNP/NPR-B signaling on bone growth, CNP or its analogs could have clinical implications for patients with impaired skeletal growth. A clinical trial of a CNP analog, vosoritide, for treatment of achondroplasia, one form of physeal skeletal dysplasia, is currently under way (ClinicalTrials.gov, NCT02055157). However, as is often the case with peptide hormones, CNP-like proteins are easily degraded or metabolized by subcutaneous neutral endopeptidase or other factors, including the clearance receptor NPR-C. As a novel strategy 
A

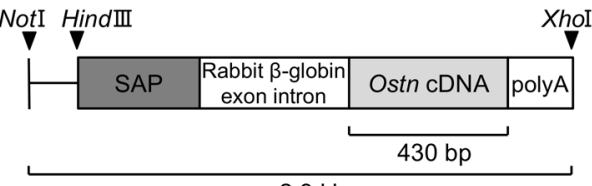

$2.3 \mathrm{~kb}$

$\mathbf{B}$

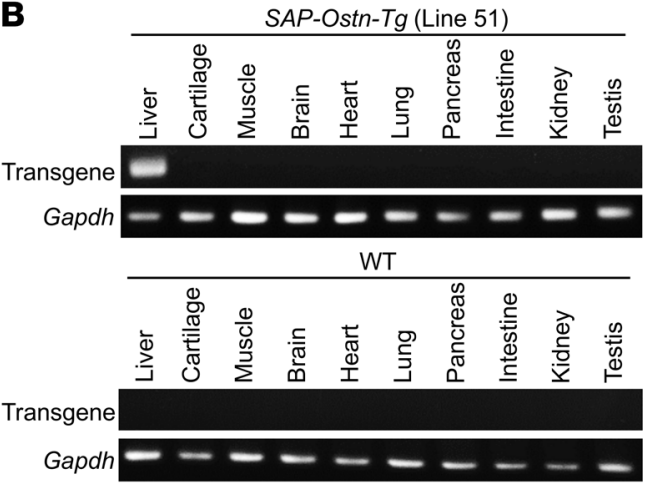

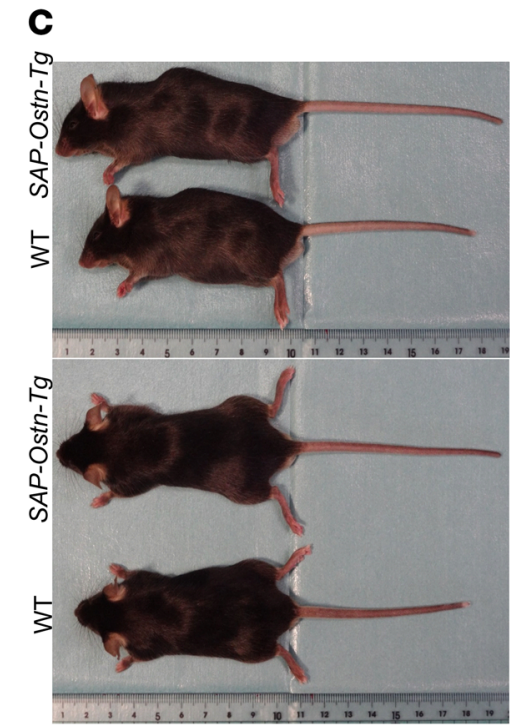

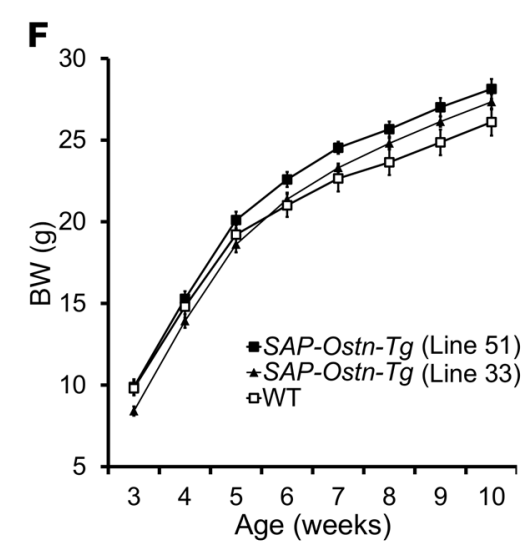

D

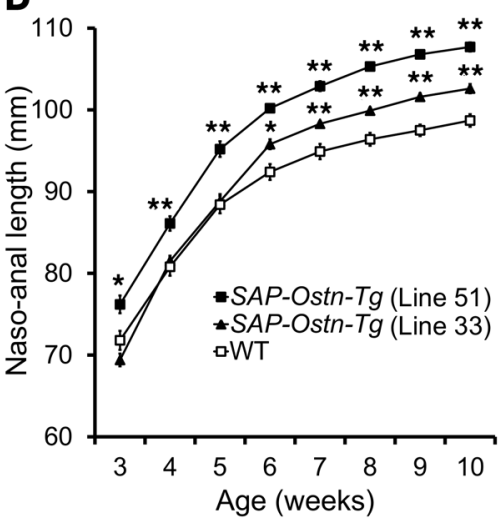

E

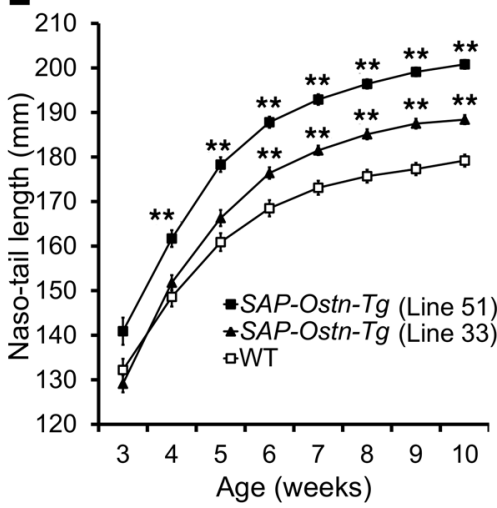

Figure 1. Generation of serum amyloid P-OSTN-transgenic (SAP-Ostn-Tg) mice. (A) Structure of the SAP-Ostn transgene. Arrowheads indicate the recognition sites of restriction endonucleases. SAP, human SAP promoter. (B) Expression of the transgene in tissues of WT and line 51 SAP-Ostn-Tg mice analyzed by RT-PCR. Transgene expression was detected only in the liver of SAP-Ostn-Tg mice. (C) Gross appearance of WT and SAP-Ostn-Tg male mice at the age of 10 weeks observed from the left side (top panel) and above (bottom panel). SAP-Ostn-Tg mice showed longer bodies, limbs, and tails than WT mice. (D-F) Growth curves based on naso-anal length (D), naso-tail length (E), and body weight (F) of WT (open squares), line 33 SAP-Ostn-Tg (filled triangles), and line 51 SAP-Ostn-Tg (filled squares) male mice measured every week from the ages of 3 to 10 weeks. Body lengths of SAP-Ostn-Tg mice became significantly larger than those of WT mice, and the body elongation of SAP-Ostn-Tg mice increased in accordance with blood OSTN levels. There was no significant difference in body weight between the 3 groups throughout the observation period. $n=10$ each. ${ }^{*} P<0.05,{ }^{*} P<0.01$ vs. WT mice, by 1 -way ANOVA followed by Tukey-Kramer test. Data are representative of 2 independent experiments (B).

for activating CNP/NPR-B signaling, we focused on modifying the clearance system of CNP by NPR-C.

Osteocrin (OSTN), also called musclin, is a small secretory peptide cloned from bone and muscle cDNA libraries in 2 independent laboratories at around the same time $(20,21)$. OSTN has a well-conserved homology with natriuretic peptide members but has no natriuretic activity. It was shown to bind NPR-C with high affinity and specificity, and could attenuate the clearance of NPR-C and increase natriuretic peptide availability $(22,23)$. In the present study, we generated transgenic mice with increased circulating levels of OSTN and examined its effects on skeletal growth. By using these transgenic mice, we elucidated the mechanism underlying the effect of OSTN on skeletal growth relevant to CNP/ NPR-B signaling by an in vivo genetic approach. Furthermore, we investigated the therapeutic potential of OSTN for reinforcing the effect of CNP on impaired skeletal growth.

\section{Results}

Generation of SAP-Ostn-Tg mice. To investigate the effect of increased circulating OSTN, we generated Ostn-transgenic mice with elevated plasma levels of OSTN due to liver-specific Ostn overexpression under the control of the human serum amyloid-P (SAP) component promoter (SAP-Ostn-Tg mice) (Figure 1A). We obtained 5 lines of SAP-Ostn-Tg mice and used heterozygous transgenic mice for all of the following experiments. Quantitative real-time PCR analysis of their genomic DNA revealed that among these lines of SAP-Ostn-Tg mice, 3 lines (lines 3, 33, and 44) had 2 copies, 1 line (line 5) had 10 copies, and the other line (line 51) had 18 copies of the transgene. Thereafter, line 33 and line 51 were used as representatives for $S A P$-Ostn- $\mathrm{Tg}$ mice with different transgene copy numbers. By using reverse transcription PCR (RT-PCR), we detected transgene expression only in the liver of SAP-Ostn-Tg mice (Figure 1B). At the age of 6 weeks, blood OSTN levels in line 
A

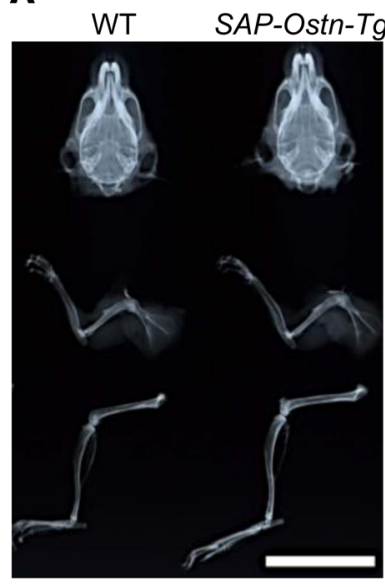

D

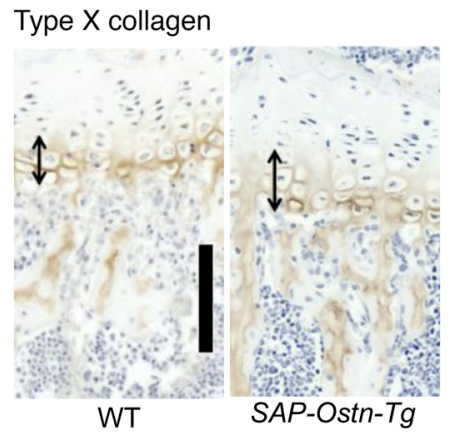

B

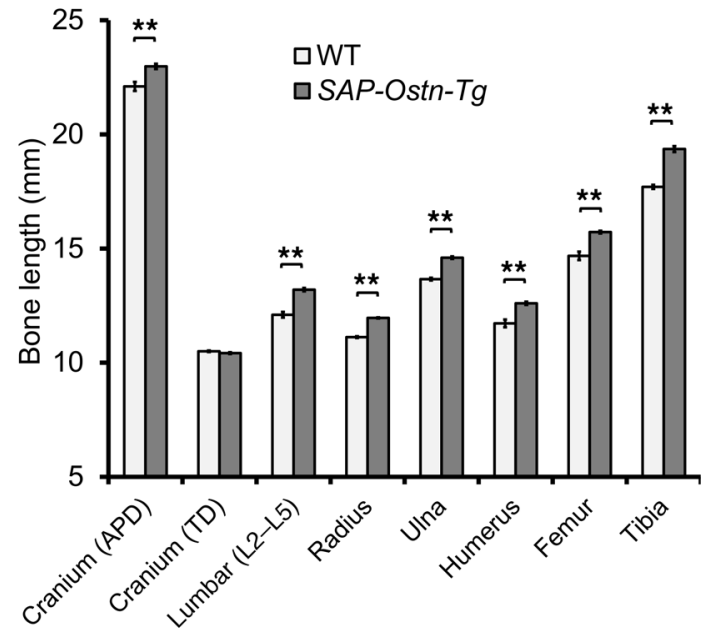

C

Alcian blue and H\&E staining

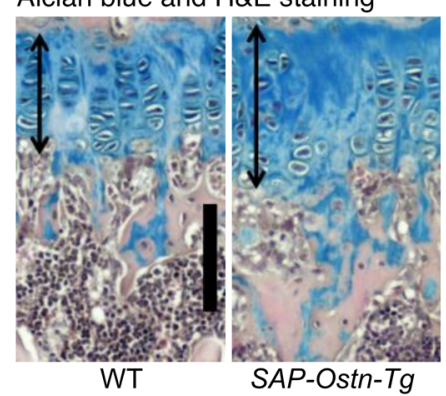

E

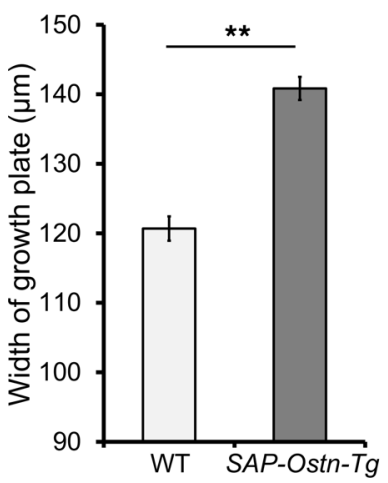

$\mathbf{F}$

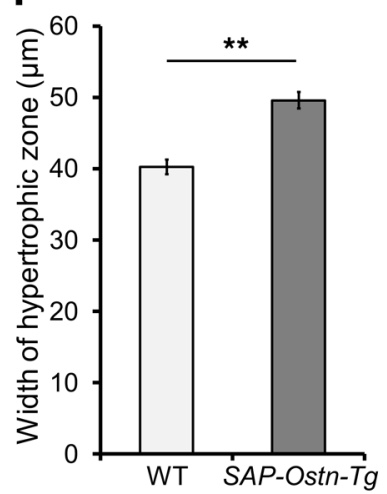

Figure 2. Skeletal phenotypes of SAP-Ostn-Tg mice. (A) Radiographs of the skulls, forelimbs, and hind limbs of WT and SAP-Ostn-Tg mice at the age of 10 weeks. SAP-Ostn-Tg mice showed skeletal overgrowth in each bone compared with WT mice. Scale bar: $2 \mathrm{~cm}$. (B) Bone lengths of WT and SAP-Ostn-Tg mice at the age of 10 weeks measured on soft $x$-ray films. Lengths of the skull, lumbar spine, radius, ulna, humerus, femur, and tibia were significantly larger in SAP-Ostn-Tg mice than in WT mice. Width of the skull was not different between the 2 genotypes. APD, anteroposterior diameter; TD, transverse diameter. $n=5$ each, for each bone length in WT and SAP-Ostn-Tg mice. (C and D) Histological pictures of the growth plates of Alcian blue and H\&E staining (C) and immunohistochemical staining for type $X$ collagen (D). Left panels show the growth plates of 10-week-old WT mice, and right panels show those of 10-week-old SAP-Ostn-Tg mice. Arrows indicate the widths of growth plates (C) and hypertrophic chondrocyte layers (D). Scale bars in C and D: 100 $\mu \mathrm{m}$. (E and $\mathbf{F}$ ) Width of growth plate $(\mathbf{E})$ and hypertrophic zone $(\mathbf{F})$ of WT and SAP-Ostn-Tg mice at the age of 10 weeks measured on the pictures.

SAP-Ostn-Tg mice had significantly thicker growth plates and hypertrophic chondrocyte layers than WT mice. $n=5$ each. ${ }^{* *} P<0.01$, by Student's $t$ test.

33 were $1.2 \mathrm{ng} / \mathrm{ml}$ (average; $n=2$ ) and those in line 51 SAP-Ost $n-T g$ mice were $52.2 \pm 20.4 \mathrm{ng} / \mathrm{ml}$ (means $\pm \mathrm{SEM} ; n=4$ ), whereas those in WT mice $(n=4)$ were below the detection limit of our measurement system, indicating that plasma OSTN concentrations were elevated in accordance with the copy number of the transgene.

Gross appearance and growth curves of SAP-Ostn-Tg mice. Pictures in Figure 1C show the gross appearance of 10-week-old male WT and line 51 SAP-Ostn-Tg mice observed from the left side and from the top. SAP-Ostn-Tg mice exhibited obviously longer bodies, paws, limbs, and tails than WT mice. The growth curves for male SAP-Ostn-Tg mice revealed that line 51 SAP-Ostn-Tg mice were significantly longer than WT mice from the age of 3 weeks by nasoanal measurement and four weeks by naso-tail measurement. The increment in body and tail lengths was less pronounced in line 33 SAP-Ostn-Tg mice than in line 51 SAP-Ostn-Tg mice throughout the observation period. Line 33 SAP-Ostn-Tg mice became significantly longer than WT mice after 6 weeks of age both in naso-anal and in naso-tail lengths (Figure 1, D and E). SAP-Ostn-Tg mice tended to be heavier than WT mice, but there was no significant difference in body weight between the 3 groups throughout the observation period (Figure 1F). Female SAP-Ostn-Tg mice had identical phenotypes to those observed for male SAP-Ostn-Tg mice, so we decided to use male mice for the following experiments (Supplemental Figure 1; supplemental material available online with this article; https://doi.org/10.1172/JCI94912DS1). As for the line of SAP-Ostn$\mathrm{Tg}$ mice, we chose to use line 51 as a representative.

Skeletal phenotype of SAP-Ostn-Tg mice. Next, we examined the skeletal phenotype of SAP-Ostn-Tg mice at the age of 10 weeks. As shown by the soft $\mathrm{x}$-ray picture of skulls viewed from the top, forelimbs, and hind limbs, we observed skeletal overgrowth in SAP-Ostn-Tg mice compared with WT mice (Figure 2A). Measuring of each bone length on the soft $\mathrm{x}$-ray film revealed that bones formed through endochondral ossification, i.e., lumbar spine, radius, ulna, humerus, femur, and tibia, were significantly longer in SAP-Ostn-Tg mice than in WT mice. The longitudinal length of the skull, which is mainly defined by endochondral ossification, 
A
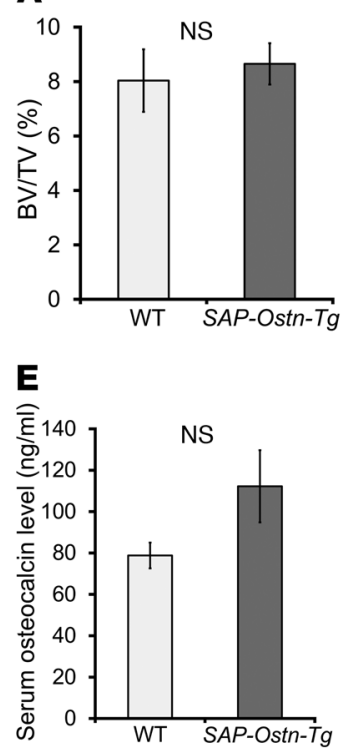

B

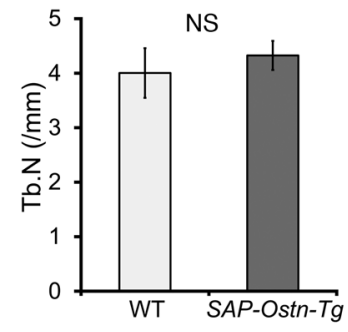

$\mathbf{F}$

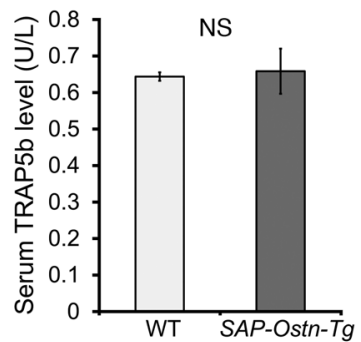

C

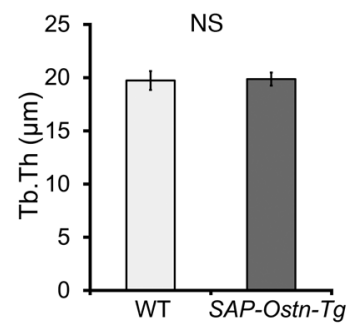

D

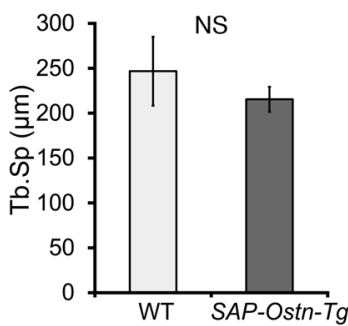

Figure 3. Analyses of bone microstructure by micro-CT and measurement of serum bone metabolic markers in SAP-Ostn-Tg mice. (A-D) Micro-CT analyses of the distal metaphyses of femurs of 10-week-old WT and SAP-Ostn-Tg mice. Graphs of bone volume fraction (BV/TV) (A), trabecular number (Tb.N) (B), trabecular thickness (Tb.Th) (C), and trabecular separation (Tb.Sp) (D) are shown. There were no differences in each parameter of bone microstructure between the 2 genotypes. $n=5$ and 6 for WT mice and SAP-Ostn-Tg mice, respectively. (E and F) Serum osteocalcin (E) and TRAP5b (F) levels of 10-weekold WT and SAP-Ostn-Tg mice. There were no significant differences in serum bone metabolic markers between the 2 genotypes. $n=4$ each. Student's $t$ test was used for statistical analysis. Data are representative of 3 independent experiments (E and $\mathbf{F})$.

was significantly greater in SAP-Ostn-Tg mice than in WT mice, whereas the skull width, which is defined by membranous ossification, was no different between the 2 genotypes (Figure 2B).

Histological analysis of the growth plate of SAP-Ostn-Tg mice. We next investigated the histology of the growth plates of SAP-Ostn-Tg mice. Histological images of the tibial growth plates of 10-weekold mice revealed that $S A P-O s t n-T g$ mice had significantly thicker growth plates than WT mice (Figure 2, C and E). The thickness of hypertrophic chondrocyte layers, as detected by the immunochemical staining for type X collagen, was larger in SAP-Ostn$\mathrm{Tg}$ mice than in WT mice (Figure 2, D and F).

Bone microstructural analysis and measurement of serum bone metabolic markers of SAP-Ostn-Tg mice. Next, we evaluated bone mass and bone metabolism of SAP-Ostn-Tg mice. We performed microstructural analysis of the distal metaphyses of femurs of 10-week-old WT and SAP-Ostn-Tg mice using micro-CT. All bone morphometric parameters, i.e., bone volume fraction, trabecular thickness, trabecular number, and trabecular separation, were no different between the 2 genotypes (Figure 3, A-D). We then measured serum levels of osteocalcin and TRAP5b as bone metabolic markers in 10-week-old WT and SAP-Ostn-Tg mice. There was no significant difference in both serum osteocalcin and TRAP5b levels between the 2 genotypes (Figure 3, E and F).

Mechanistic analyses of skeletal overgrowth in SAP-Ostn-Tg mice. OSTN has been reported to be a selective ligand for NPR-C and to attenuate the clearance of this receptor, resulting in an increase in natriuretic peptide availability $(22,23)$. Among the natriuretic peptides, CNP is known to be a strong stimulator of endochondral bone growth, so we hypothesized that the skeletal overgrowth phenotype observed in SAP-Ostn-Tg mice is due mainly to the increased availability of CNP through the occupation of NPR-C by increased circulating OSTN. To test this hypothesis, we generated SAP-Ostn-Tg mice depleted of CNP ( $\mathrm{Nppc}^{-/} / \mathrm{SAP}-\mathrm{Ostn-Tg} \mathrm{mice)}$ and compared their growth and skeletal phenotype with those of CNP-knockout mice ( $N p p c^{-/-}$mice). At a glance, $N p p c^{-/-} / S A P-O s t n-$ $\mathrm{Tg}$ mice had the same appearance as $\mathrm{Np}^{-1 /}$ mice (Figure $4 \mathrm{~A}$ ). As shown by the growth curves, there were no significant differences in naso-anal and naso-tail lengths and body weight between $\mathrm{Nppc}^{-1-}$ / SAP-Ostn-Tg mice and Nppc $\mathrm{C}^{-/}$mice (Figure 4B and Supplemental Figure 2, A and B). Furthermore, at the age of 9 weeks, the length of each bone and the thickness of tibial growth plates in $N p p c^{-1-} / S A P-$ $\mathrm{Ostn-Tg}$ mice were equal to those in $\mathrm{Nppc}^{-/-}$mice (Figure 4, C-E).

Next, to confirm whether the skeletal overgrowth observed in SAP-Ostn-Tg mice is mediated by NPR-C, we generated SAP-Ostn$\mathrm{Tg}$ mice depleted of NPR-C (Npr3--/SAP-Ostn-Tg mice) and compared their growth and skeletal phenotypes with those of NPR-Cknockout mice ( $\mathrm{Npr}^{-/-}$mice). The growth curves for $\mathrm{Npr}^{-1-} / \mathrm{SAP}$ Ostn-Tg mice were similar to those for $\mathrm{Npr}^{-/-}$mice (Figure $4 \mathrm{~F}$ and Supplemental Figure 2, C and D). At the age of 10 weeks, each bone length and the thickness of tibial growth plates in $\mathrm{Npr}^{-1-} / \mathrm{SAP}$-Ostn$\mathrm{Tg}$ mice were equivalent to those in $\mathrm{Npr}^{-/-}$mice (Figure 4, G-I).

In addition, we measured plasma CNP concentrations in 6-weekold WT and SAP-Ostn-Tg mice. We thereby determined that SAPOstn-Tg mice had significantly higher blood CNP levels $(1.31 \pm 0.23$ $\mathrm{pM}, n=4, P<0.01)$ than WT mice $(0.41 \pm 0.05 \mathrm{pM}, n=4)$ (Figure $4 \mathrm{~J})$.

We analyzed Nppc (CNP), Npr3 (NPR-C), and Npr2 (NPR-B) mRNA expression in epiphyseal cartilage of tibiae and lumbar vertebrae of 3-week-old WT and SAP-Ostn-Tg mice. In tibial cartilage and lumbar vertebrae, there were no significant differences in both $N p p c$ and Npr2 mRNA expression levels between the 2 
A
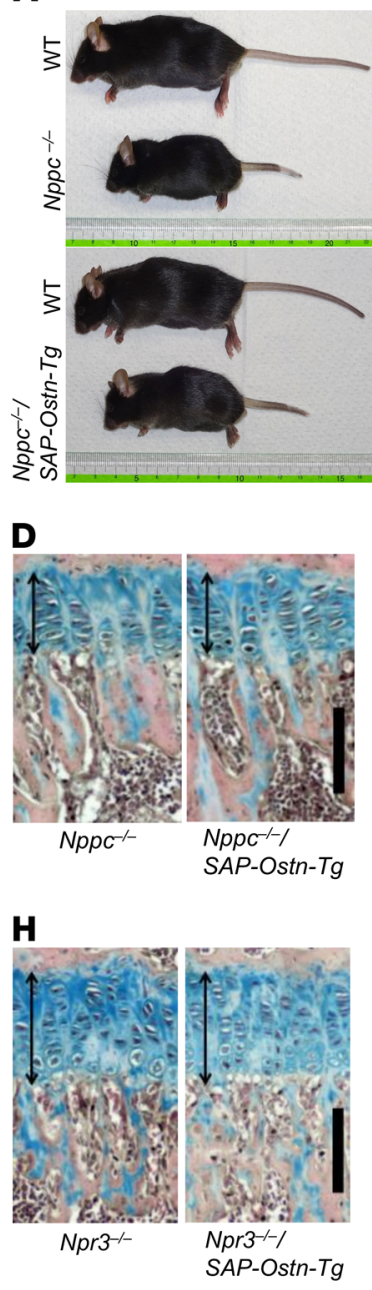

B
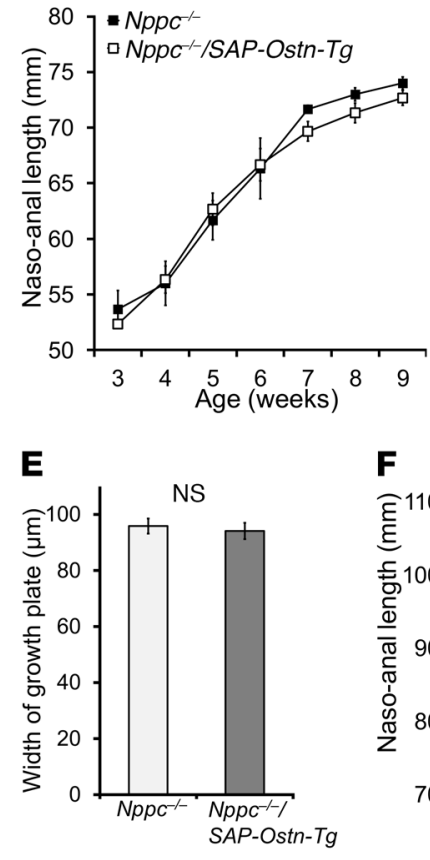

I

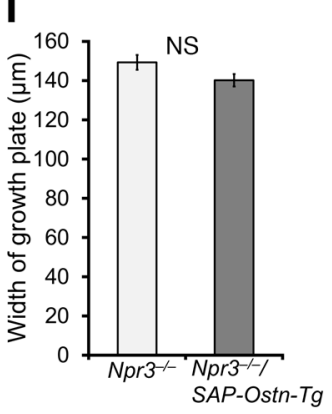

\section{J}

C

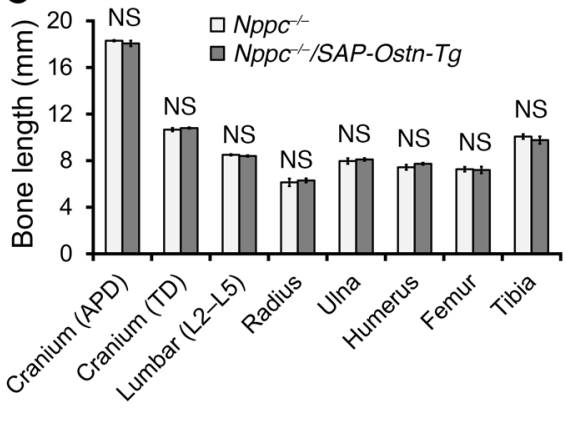

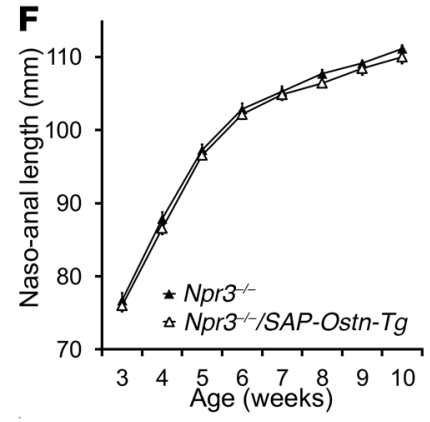

G

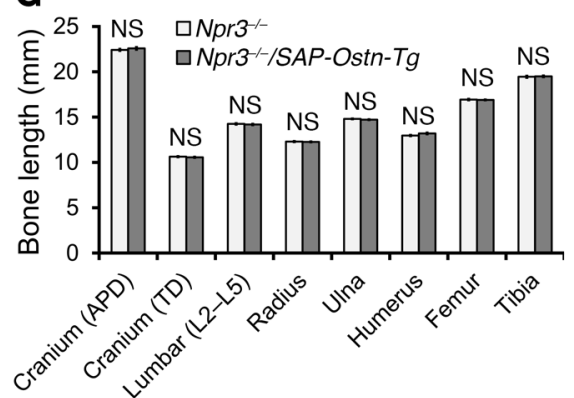

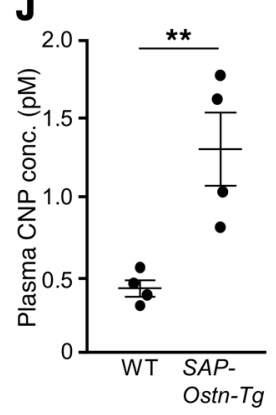

Figure 4. Mechanistic analyses of skeletal overgrowth in SAP-Ostn-Tg mice. (A) Gross appearance of $\mathrm{Nppc}^{-/-}$and Nppc/-/SAP-Ostn-Tg mice. Top panel shows 9-week-old WT and $\mathrm{Nppc}^{-/-}$mice, and bottom panel shows 9-week-old WT and Nppc-//SAP-Ostn-Tg mice. (B) Growth curves of naso-anal length of $\mathrm{Nppc}^{-/-}$(filled squares) and $\mathrm{Nppc}^{-/-} / \mathrm{SAP}-\mathrm{Ostn}-\mathrm{Tg}$ (open squares) mice measured every week from the ages of 3 to 9 weeks. $n=3$ each. (C) Bone lengths of 9-week-old $\mathrm{Nppc}^{-/-}$and $\mathrm{Nppc}^{-/-} / \mathrm{SAP}$-Ostn-Tg mice. $\mathrm{n}=3$ each, for each bone length in $\mathrm{Nppc}^{-/-}$and $\mathrm{Nppc}^{-1 /} / \mathrm{SAP}-\mathrm{Ostn}-\mathrm{Tg}$ mice. (D) Histological photographs of the tibial growth plates of Alcian blue and H\&E staining of 9-week-old $\mathrm{Nppc}^{-/-}$and $\mathrm{Nppc}^{-1-} / \mathrm{SAP}-\mathrm{Ostn}-\mathrm{Tg}$ mice. Arrows indicate the widths of growth plates. Scale bar: $100 \mu \mathrm{m}$. (E) The widths of the tibial growth plates of 9-week-old $N p p c^{-/-}$and $N p p c^{-1-/ S A P-O s t n-T g ~ m i c e . ~} n=3$ each. (F) Growth curves of naso-anal length of $\mathrm{Npr}^{-1-}$ (filled triangles) and $\mathrm{Npr}^{-1-} / \mathrm{SAP}-\mathrm{Ostn}-\mathrm{Tg}$ (open triangles) mice measured every week from the ages of 3 to 10 weeks. $n=7$ each. (C) Bone lengths of 10-week-old $\mathrm{Npr}^{-/-}$and $\mathrm{Npr} 3^{-/-} / \mathrm{SAP}$-Ostn-Tg mice. $n=6$ each, for each bone length in $\mathrm{Npr}^{-/-}$and $\mathrm{Npr} 3^{-/-} / \mathrm{SAP}-\mathrm{Ostn}-\mathrm{Tg}$ mice. (H) Histological images of the tibial growth plates of Alcian blue and H\&E staining of 10 -week-old $\mathrm{Npr}^{-1 /-}$ and $\mathrm{Npr}^{-1-} / \mathrm{SAP}-\mathrm{Ostn}$-Tg mice. Arrows indicate the widths of

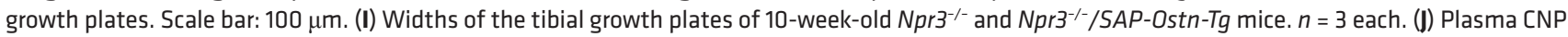
concentrations of 6-week-old WT and SAP-Ostn-Tg mice. $n=4$ each. ${ }^{* *} P<0.01$, by Student's $t$ test.

genotypes. On the other hand, the Npr3 mRNA expression level in $S A P-O s t n-T g$ mice tended to be higher in tibial cartilage and was significantly higher in lumbar vertebrae than that in WT mice (Figure 5 and Supplemental Figure 3).

Additive effect of CNP and OSTN on the skeletal growth in doubletransgenic mice. We previously reported that CNP-transgenic mice with an increased circulating CNP under the control of the SAP promoter (SAP-Nppc-Tg mice) exhibit a prominent skeletal overgrowth phenotype (5). To investigate whether OSTN could augment this effect of CNP, we generated double-transgenic mice with increased circulating CNP levels as well as OSTN by crossing $S A P-N p p c-T g$ mice with SAP-Ostn-Tg mice (SAP-Nppc-Tg/SAPOstn-Tg mice). Photographs of the gross appearance of mice at the age of 10 weeks showed that $S A P-N p p c-T g$ mice and $S A P$ Ostn-Tg mice were obviously longer than WT mice, and $S A P$ $N p p c-T g / S A P-O s t n-T g$ mice were longer than both $S A P-N p p c-T g$ and SAP-Ostn-Tg mice (Figure 6A).

The growth curves showed that $S A P-N p p c-T g$ mice became significantly longer than WT mice from the age of 5 weeks in nasoanal length and 6 weeks in naso-tail length. SAP-Ostn-Tg mice were significantly longer than $S A P-N p p c-T g$ mice at earlier ages (5-6 weeks in naso-anal length and 3-9 weeks in naso-tail length), but the differences became trivial and eventually diminished from the age of 7 weeks in naso-anal length and 10 weeks in naso-tail length. SAP-Nppc-Tg/SAP-Ostn-Tg mice were significantly longer than SAP-Ostn-Tg mice, in terms of both naso-anal and naso-tail 
Tibial cartilage
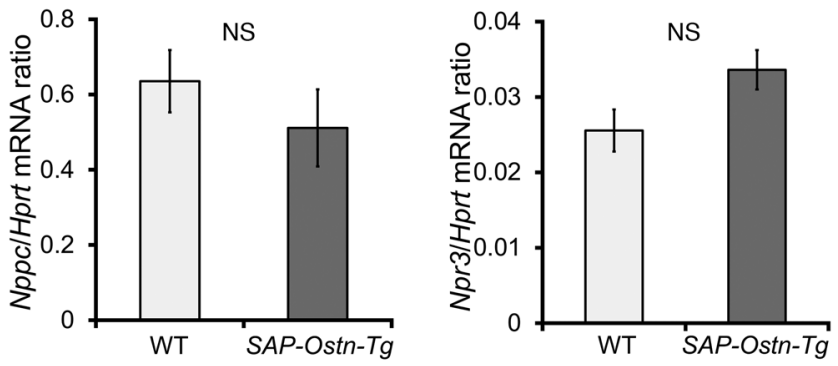

Lumbar vertebrae

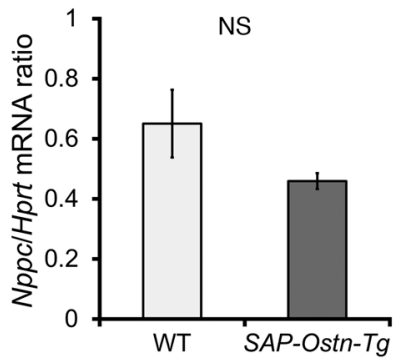

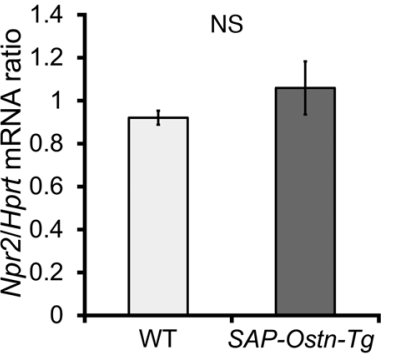

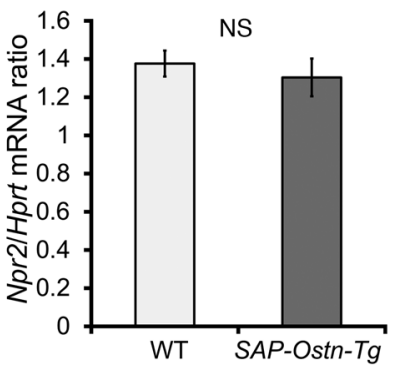

Figure 5. mRNA expression of genes in WT and SAP-Ostn-Tg mice at 3 weeks of age. mRNA expression of Nppc (CNP), Npr3 (NPR-C), and Npr2 (NPR-B) in epiphyseal cartilage of tibiae and lumbar vertebrae of 3-week-old WT and SAP-Ostn-Tg mice. Target gene mRNA expression levels were assessed by quantitative RT-PCR. Data were normalized to hypoxanthine guanine phosphoribosyl transferase (Hprt) mRNA levels. $n=5$ each in the analysis of tibial cartilage; $n=4$ each in the analysis of lumbar vertebrae. ${ }^{*} P<0.05$, by Student's $t$ test. Data are representative of at least 2 independent experiments. length from the age of 6 weeks (Figure 6, B and C). There were no significant differences in body weight among these 4 genotypes throughout the observation period (Supplemental Figure 4).

Measurement of bone lengths on soft x-ray films at the age of 10 weeks revealed that the skull, lumbar spine, radius, ulna, humerus, femur, and tibia were significantly longer in SAP-Nppc$\mathrm{Tg}$ mice than in WT mice. Moreover, those same bones, except the skull, were significantly longer in $S A P-N p p c-T g / S A P-O s t n-T g$ mice than in $S A P-N p p c-T g$ mice. The skull length of $S A P-N p p c-T g / S A P-$ $O s t n-T g$ mice tended to be greater than that of $S A P-N p p c-T g$ mice, but the difference was not significant. Each bone in the extremities, i.e., radius, ulna, humerus, femur, and tibia, was significantly longer in SAP-Nppc-Tg mice than in SAP-Ostn-Tg mice, whereas there were no differences in skull and lumbar spine lengths between the 2 genotypes. Skull widths were no different between the 4 genotypes (Figure 6D and Table 1).

Histological analysis of the tibial growth plates of 10-weekold mice showed that $S A P-N p p c-T g$ mice had significantly thicker growth plates than SAP-Ostn-Tg mice, and SAP-Nppc-Tg/SAP-Ostn$\mathrm{Tg}$ mice had significantly thicker growth plates than $S A P-N p p c-T g$ mice (Figure 6, E and G). The thickness of hypertrophic chondrocyte layers positive for type $\mathrm{X}$ collagen immunostaining was significantly greater in $S A P-N p p c-T g$ mice than in SAP-Ostn-Tg mice and even greater in SAP-Nppc-Tg/SAP-Ostn-Tg mice (Figure 6, $\mathrm{F}$ and $\mathrm{H}$ ).

Next, we measured plasma CNP concentrations of 6-weekold mice and found no significant difference in plasma CNP levels between SAP-Ostn-Tg and SAP-Nppc-Tg mice $(1.31 \pm 0.23 \mathrm{pM}$ and $1.79 \pm 0.12 \mathrm{pM}, n=4 \mathrm{each})$. We also found that $S A P-N p p c-$ $\mathrm{Tg} / \mathrm{SAP}$-Ostn-Tg mice had significantly higher blood CNP levels $(4.25 \pm 0.82 \mathrm{pM}, n=4, P<0.01)$ than $S A P-O s t n-T g$ and $S A P$ $\mathrm{Nppc-Tg} \mathrm{mice} \mathrm{(Figure} \mathrm{6I).}$

There were some differences in the growth rates or body proportions between SAP-Ostn-Tg mice and SAP-Nppc-Tg mice despite their comparable plasma CNP concentrations at 6 weeks of age. To elucidate the mechanisms underlying these differences, we analyzed $N p p c, N p r 3$, and Npr2 mRNA expressions in epiphyseal cartilage of tibiae, lumbar vertebrae, and blood vessels of 3- and 6-week-old WT mice. In tibial cartilage, the Nppc mRNA expression level in 3-week-old mice tended to be higher than that in 6-week-old mice, while the Npr3 mRNA expression level of

Table 1. Statistical differences in the length of each bone between WT, SAP-Ostn-Tg, SAP-Nppc-Tg, and SAP-Ostn-Tg/SAP-Nppc-Tg mice

\begin{tabular}{|c|c|c|c|c|c|c|c|c|}
\hline & \multicolumn{2}{|c|}{ Cranium } & \multirow[t]{2}{*}{ Lumbar } & \multirow[t]{2}{*}{ Radius } & \multirow[t]{2}{*}{ Ulna } & \multirow[t]{2}{*}{ Humerus } & \multirow[t]{2}{*}{ Femur } & \multirow[t]{2}{*}{ Tibia } \\
\hline & APD & TD & & & & & & \\
\hline SAP-Nppc-Tg vs. SAP-Nppc-Tg/SAP-Ostn-Tg & NS & NS & $P<0.01$ & $P<0.01$ & $P<0.05$ & $P<0.01$ & $P<0.01$ & $P<0.01$ \\
\hline SAP-Ostn-Tg vs. SAP-Nppc-Tg/SAP-0stn-Tg & NS & NS & $P<0.01$ & $P<0.01$ & $P<0.01$ & $P<0.01$ & $P<0.01$ & $P<0.01$ \\
\hline WT vs. SAP-Nppc-Tg/SAP-Ostn-Tg & $P<0.01$ & NS & $P<0.01$ & $P<0.01$ & $P<0.01$ & $P<0.01$ & $P<0.01$ & $P<0.01$ \\
\hline WT vs. SAP-Nppc-Tg & $P<0.05$ & NS & $P<0.01$ & $P<0.01$ & $P<0.01$ & $P<0.01$ & $P<0.01$ & $P<0.01$ \\
\hline
\end{tabular}

Significance was determined by 1-way ANOVA followed by Tukey-Kramer test. APD, anteroposterior diameter; TD, transverse diameter. 
A

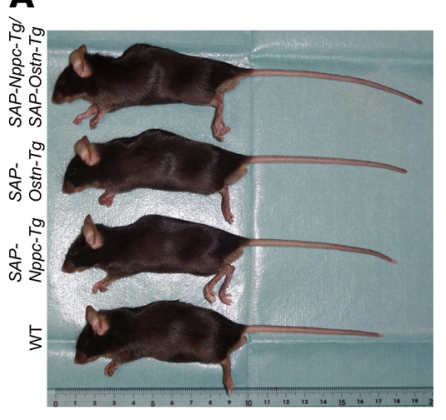

B

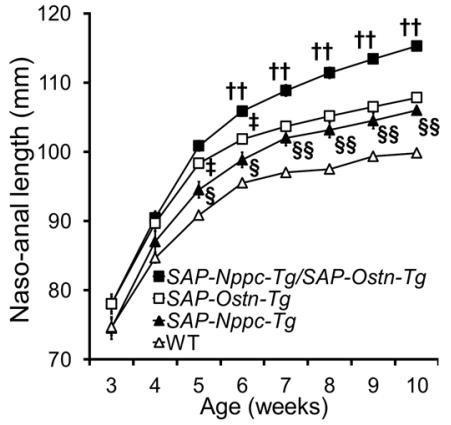

E
D

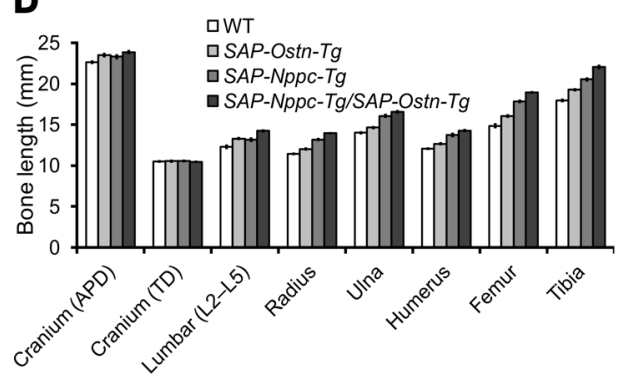

Alcian blue and H\&E staining

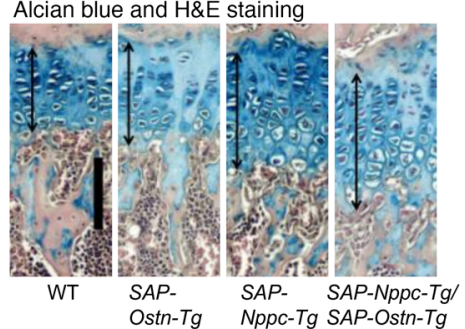

C

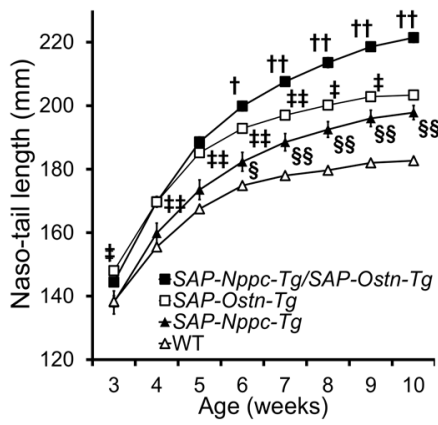

$\mathbf{F}$

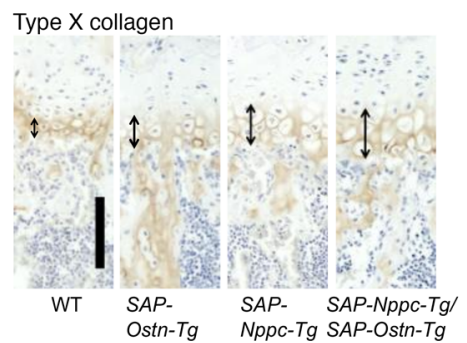

G

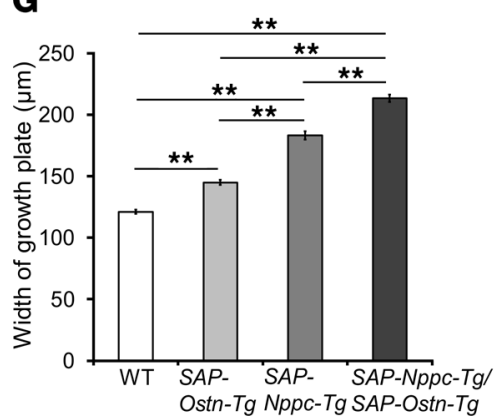

H

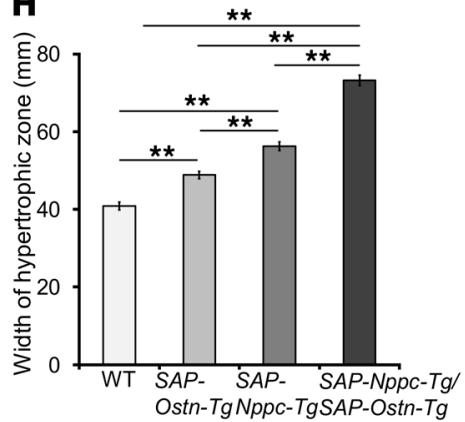

I

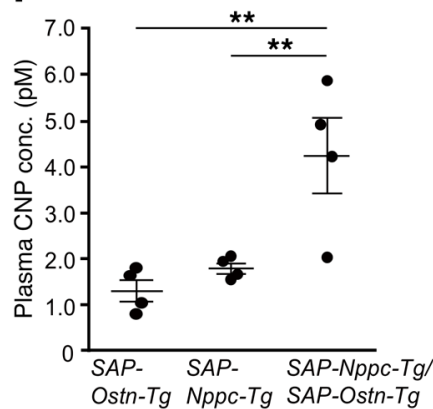

Figure 6. Additive effect of CNP and OSTN on the skeletal growth in double-transgenic mice. (A) Gross appearance of 10-week-old WT, SAP-Nppc-Tg, SAP-Ostn-Tg, and SAP-Nppc-Tg/SAP-Ostn-Tg mice. SAP-Nppc-Tg/SAP-Ostn-Tg mice were longer than SAP-Nppc-Tg and SAP-Ostn-Tg mice. (B and C) Growth curves based on naso-anal (B) and naso-tail length (C) of WT (open triangles), SAP-Nppc-Tg (filled triangles), SAP-Ostn-Tg (open squares), and SAP-Nppc-Tg/SAP-Ostn-Tg (filled squares) mice measured every week from the ages of 3 to 10 weeks. $n=6,6,6$, and 7 in WT, SAP-Nppc-Tg, SAP-Ostn-Tg, and SAP-Nppc-Tg/SAP-Ostn-Tg mice, respectively. ${ }^{\dagger} P<0.05,{ }^{\dagger \dagger} P<0.01$ vs. SAP-Ostn-Tg mice; ${ }^{\ddagger} P<0.05,{ }^{\ddagger \ddagger} P<0.01$ vs. SAP-Nppc-Tg mice; ${ }^{\S} P<0.05$, $\$ \$ P<0.01$ vs. WT mice. One-way ANOVA followed by Tukey-Kramer test was used for statistical analysis. (D) Bone lengths of 10-week-old WT, SAPNppc-Tg, SAP-Ostn-Tg, and SAP-Nppc-Tg/SAP-Ostn-Tg mice. $n=6,6,6$, and 7 in WT, SAP-Nppc-Tg, SAP-Ostn-Tg, and SAP-Nppc-Tg/SAP-Ostn-Tg mice, respectively. Statistical differences between genotypes are summarized in Table 1. (E and F) Histological pictures of the growth plates of 10-week-old mice. Alcian blue and H\&E staining (E) and immunohistochemical staining for type $X$ collagen $(\mathbf{F})$ are shown. Arrows indicate the widths of growth plates (E) and hypertrophic chondrocyte layers (F). Scale bars in $\mathbf{E}$ and $\mathbf{F}: 100 \mu \mathrm{m}$. (G and $\mathbf{H})$ Widths of growth plates $(\mathbf{C})$ and hypertrophic zone (H) of 10-week-old mice. $n=6,6,6$, and 7 in WT, SAP-Nppc-Tg, SAP-Ostn-Tg, and SAP-Nppc-Tg/SAP-Ostn-Tg mice, respectively. (I) Plasma CNP concentrations in 6-week-old SAPOstn-Tg, SAP-Nppc-Tg, and SAP-Nppc-Tg/SAP-Ostn-Tg mice. There was no significant difference in plasma CNP levels between SAP-Ostn-Tg and SAP-Nppc-Tg mice. SAP-Nppc-Tg/SAP-Ostn-Tg mice had significantly higher blood CNP levels than SAP-Ostn-Tg and SAP-Nppc-Tg mice. $n=4$ each. ${ }^{* *} P<0.01$, by 1-way ANOVA followed by Tukey-Kramer test.

3-week-old mice was unchanged compared with that of 6-weekold mice. In lumbar vertebrae, the Nppc mRNA expression level of 3-week-old mice was significantly higher than that of 6-weekold mice, and the Npr3 mRNA expression level of 3-week-old mice did not differ significantly from that of 6-week-old mice. In blood vessels, the Nppc mRNA expression level was higher and the Npr3 mRNA expression level was lower in 3-week-old mice than in 6-week-old mice, but the differences were not significant. In all the tissues examined, there were no significant differences in the Npr2 mRNA expression levels between 3-week-old and 6-weekold mice (Figure 7 and Supplemental Figure 5).
In addition, we measured cGMP levels of lumbar vertebrae of 3-week-old WT, SAP-Nppc-Tg, and SAP-Ostn-Tg mice. There were no significant differences in vertebral cGMP levels between the 3 genotypes, but those of $S A P-N p p c-T g$ and SAP-Ostn-Tg mice, especially that of $S A P-O s t n-T g$ mice, tended to be higher than that of WT mice (Figure 8).

\section{Discussion}

In general, bioactive peptides are safe and useful remedies for treating diseases, but they are often easily degraded or metabolized by endopeptidases or some other factors. Besides developing analogs 

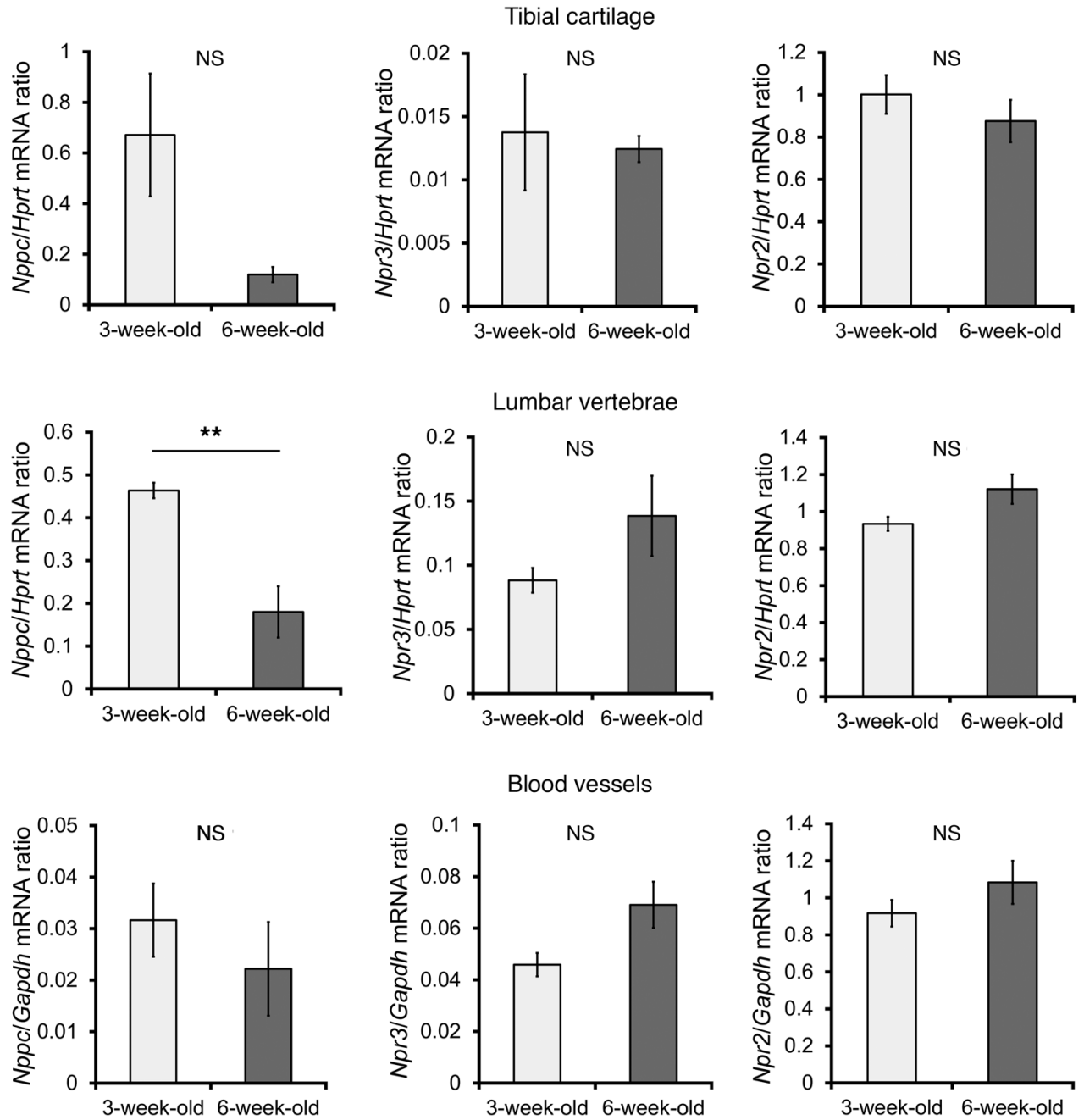

Figure 7. mRNA expression of genes in WT mice at $\mathbf{3}$ and $\mathbf{6}$ weeks of age. mRNA expression of Nppc, Npr3, and Npr2 in epiphyseal cartilage of tibiae, lumbar vertebrae, and blood vessels of 3- and 6-week-old WT mice. Data from tibial cartlilages and lumbar vertebrae were normalized to Hprt mRNA levels. Data from blood vessels were normalized to Gapdh mRNA levels. $n=4$ each. ${ }^{* *} P<0.01$, by Student's $t$ test. Data are representative of at least 2 independent experiments.

resistant to degradation, dampening the degradation or clearance systems for peptide ligands is a promising strategy for increasing the efficacy of peptide therapies. We have been engaged in translational research concerning CNP/NPR-B signaling in impaired skeletal growth (4). In the case of CNP/NPR-B signaling, the endogenous ligand, CNP, is easily degraded by neutral endopeptidase or cleared by NPR-C. As OSTN was shown to be a specific ligand for NPR-C (20), we examined the effect of circulating OSTN on skeletal growth and investigated the mechanism relevant to the effect of CNP/NPR-B signaling. Whereas Moffatt et al. suggested that the stimulatory effect of OSTN on skeletal growth is the augmentation of CNP by OSTN through an in vitro biochemical approach by showing increased levels of the second messenger cGMP in chondrogenic and osteoblastic cell lines (22), we tried to elucidate the mechanism by an in vivo genetic approach using transgenic and knockout mice. At first, plasma CNP concentrations were elevated in SAP-Ostn-Tg mice, suggesting that the elongation of bones in SAP-Ostn-Tg mice is due at least in part to an increase in circulating CNP. Second, the fact that the growth-stimulating effect of OSTN was completely eliminated by CNP depletion clearly demonstrates that CNP is indispensable for this effect. Third, the lack of further elongation by OSTN in NPR-C-knockout mice shows that NPR-C is necessary for OSTN to exert its stimulatory effect on bone growth.

Because local CNP as well as NPR-B in the growth plate cartilage is essential for endochondral bone growth as indicated by the result of impaired skeletal growth in cartilage-specific Nppcor Npr2-knockout mice (24), the OSTN-induced stimulation of endochondral bone growth in SAP-Ostn-Tg mice is deduced to be caused by increased OSTN-binding to local NPR-C in the growth plate cartilage. Although we have FACS data that suggest the binding of OSTN to NPR-C in a chondrogenic cell line, ATDC5 (Haruko Watanabe-Takano et al., unpublished observations), we could not actually prove that the elevated OSTN indeed binds to NPR-C in vivo in the cartilage of SAP-Ostn-Tg mice; to show and evaluate the binding of OSTN to NPR-C in vivo in the cartilage is technically difficult. Accordingly, we must take into consideration that our present study does not completely rule out an effect of OSTN that is independent of 


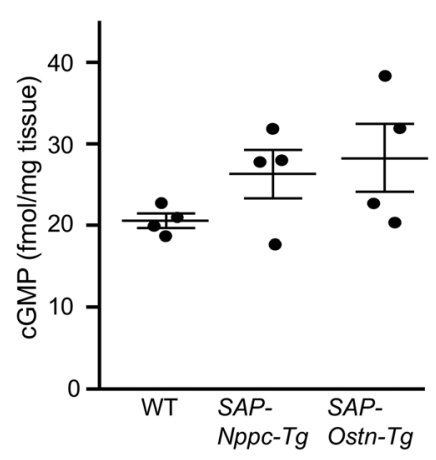

Figure 8. cGMP levels in lumbar vertebrae of 3 -week-old WT, SAP-Nppc-Tg, and SAPOstn-Tg mice. cGMP levels in lumbar vertebrae of 3-week-old mice were measured by ELISA. $n=4$ each. One-way ANOVA followed by Tukey-Kramer test was used for statistical analysis. Data are representative of 3 independent experiments

NPR-C, even in spite of the result of the crossing experiment of SAP-Ostn-Tg and NPR-C-knockout mice.

As for alteration of expression in CNP, NPR-C, and NPR-B by increased OSTN in SAP-Ostn-Tg mice, the NPR-C mRNA level in the cartilage increased in SAP-Ostn-Tg mice compared with WT mice. The reason is not known, but the increased occupation of NPR-C by OSTN might upregulate the expression of NPR-C itself. On the other hand, the expression of CNP might have a tendency to decrease in SAP-Ostn-Tg mice, suggesting the existence of local negative-feedback regulation of CNP in cartilage. Nevertheless, the precise regulatory mechanism of the CNP/NPR-B system including OSTN/NPR-C in cartilage has not yet been fully studied, and the investigation is now ongoing in our laboratory.

Taking clinical settings into consideration, it is important that OSTN exerts growth-stimulating effects on bones in a different manner from CNP in vivo. According to the results of the crossing experiment involving SAP-Ostn-Tg and CNP-knockout mice in this study, the stimulatory effect on bone growth in SAP-Ostn-Tg mice is due to intrinsic CNP production. Although SAP-Ostn-Tg mice and $S A P-N p p c-T g$ mice were generated using the same SAP promoter that causes their respective transgenes to be expressed just after birth (25), there is a fundamental difference in that the stimulatory effect on bone growth in SAP-Ostn-Tg mice depends on enhanced intrinsic CNP production, whereas that in SAP-Nppc-Tg mice is due to de novo production of CNP by the transgene.

The growth curves for SAP-Ostn-Tg and SAP-Nppc-Tg mice revealed that $S A P-O s t n-T g$ mice were longer than $S A P-N p p c-T g$ mice at early ages and later became equal to $S A P-N p p c-T g$ mice in length, indicating that the growth-stimulating effect on axial bones was stronger in SAP-Ostn-Tg mice than in SAP-Nppc-Tg mice at early ages. This suggests that CNP contents in vertebrae were larger in SAP-Ostn-Tg mice than in SAP-Nppc-Tg mice at early ages. This estimation is supported by the result that cGMP contents, the second messenger of CNP/NPR-B signaling, in vertebrae of 3-weekold SAP-Ostn-Tg mice tended to be larger than those in vertebrae of SAP-Nppc-Tg mice, and it is further supported by the result that CNP expression levels in vertebrae were significantly higher in 3-week-old mice than in 6-week-old mice, whereas NPR-C and NPR-B expression levels were unchanged between 3- and 6-weekold mice. Besides, the measurement of plasma CNP concentrations of mice at the age of 6 weeks revealed that SAP-Ostn-Tg mice had almost equal or rather lower blood CNP levels compared with $S A P-N p p c-T g$ mice, although both naso-anal and naso-tail lengths at that age were significantly greater in SAP-Ostn-Tg mice than in
SAP-Nppc-Tg mice. Most circulating CNP is thought to originate from cartilage and vessels (26). Serial expression patterns of CNP in blood vessels were identical to those in tibial cartilage and vertebrae. So, it could be assumed that WT mice as well as SAP-Ostn-Tg mice at 3 weeks of age have higher blood CNP levels than those at 6 weeks of age, although we could barely measure blood CNP levels of 3-week-old mice directly because of their small size. This notion is compatible with the fact that SAP-Ostn-Tg mice were longer than $S A P-N p p c-T g$ mice at early ages. Furthermore, the measurement of each bone length at the age of 10 weeks revealed that appendicular bones were significantly longer in SAP-Nppc-Tg mice than in SAPOstn- $\mathrm{Tg}$ mice, whereas vertebral lengths were no different between the 2 genotypes, indicating that a more potent effect on appendicular bone growth was observed in SAP-Nppc-Tg mice than in SAPOstn- $\mathrm{Tg}$ mice, especially at later ages. This may be explained by the serial changes of CNP expression levels in cartilage and by the difference of the NPR-C expression levels between tibial cartilage and vertebrae. In SAP-Ostn-Tg mice, both axial and appendicular bones would grow substantially at early ages because of a large amount of CNP in cartilage, but the rate of bone growth would decline with time in proportion to the decrease of CNP contents. On the other hand, in SAP-Nppc-Tg mice, these bones would continue to grow at an accelerated pace due to constant delivery of CNP to cartilage. Moreover, the results of quantitative PCR analysis suggest that the NPR-C expression level was much higher in vertebrae than in tibial cartilage (about 0.1 vs. 0.01, respectively, for Npr3/Hprt mRNA ratio, as shown in Figure 7), so the bone stimulatory effect of CNP, especially in SAP-Nppc-Tg mice, would be stronger in appendicular bones than in axial bones, resulting in long limbs out of proportion to the body length as observed in 10-week-old mice. Collectively, increased circulating OSTN can enhance skeletal growth, especially that of axial bones, at early ages. On the other hand, continuously increased circulating CNP stimulates appendicular bone growth more potently than OSTN, especially at later ages.

In clinical settings for the treatment of impaired skeletal growth, we could use OSTN and CNP for different purposes. Concerning therapeutic application for specific skeletal dysplasias, OSTN therapy, rather than CNP therapy, may be beneficial for the heterozygous NPR 2 defects, because they mainly cause short stature with mild skeletal defects in appendicular bones $(12,15)$. Furthermore, heterozygous NPPC defects, which have recently been identified and are associated with skeletal abnormalities similar to those seen with heterozygous NPR 2 defects, could also be a good application for OSTN therapy (16). Nevertheless, the clinical phenotype of individuals with heterozygous NPR2 defects is reported to be highly variable: some have short stature, whereas others have normal stature but shortened arms (15). Accordingly, it would be hard to generalize which therapy, i.e., OSTN or CNP, is optimal for treating patients with heterozygous NPR2 or possibly NPPC defects, and we might have to choose pertinent therapy for each patient. As for other clinical features besides growth in patients with mutations in the NPR-B/ CNP/cGMP pathway, OSTN therapy would not worsen or even improve them, because OSTN therapy could restore the impaired signaling of this pathway in situ in the whole body. On the contrary, a therapeutic approach that directly activates NPR-B by using CNP or its analog may achieve greater pharmacological efficacy for appendicular bones, so it may be suitable for treating short-limbed dwarf- 
ism such as the FGFR3 chondrodysplasia group, including achondroplasia and hypochondroplasia.

The additive effect of CNP and OSTN on the stimulation of endochondral bone growth is favorable considering simultaneous administration of these 2 molecules or their respective analogs in clinical settings. It has been speculated that increased CNP levels in $S A P-N p p c-T g$ mice were further augmented by the decrease in clearance capacity of NPR-C caused by OSTN.

As for a complication of OSTN or OSTN-CNP combination therapy, we must take decreased blood pressure into consideration, due mainly to increased atrial natriuretic peptide (ANP) and brain natriuretic peptide (BNP) actions (27). Although we have yet to check the blood pressure of our transgenic mice, we believe that this problem is not very serious considering the previously reported result on NPR-C-knockout mice, in that some degrees of increase in plasma ANP levels were observed with a slight decrease in their blood pressure (27). Nonetheless, we plan to examine the cardiovascular phenotypes, including blood pressure, of our transgenic mice in future.

In conclusion, we have elucidated the stimulatory effect of circulating OSTN on bone growth and determined mechanism by using an in vivo genetic approach in mice. Furthermore, we have confirmed the additive effect of OSTN and CNP on bone growth. In clinical settings, we will have to perform exogenous administration of OSTN or its analog, and the preclinical examination investigating the effect of OSTN administration to model animals is now ongoing in our laboratory.

\section{Methods}

Animals. C57BL/6 mice used in this study were purchased from Japan SLC, Inc.. To generate Ostn-transgenic mice, a transgene was designed to express Ostn specifically in the liver under control of the human serum amyloid-P (SAP) component promoter. A 410bp cDNA corresponding to mouse Ostn coding sequence (GenBank accession no. NM_198112.2) was synthesized with an $M f e$ I restriction site added to the $5^{\prime}$ end and an EcoRI restriction site to the $3^{\prime}$ end. This synthesized mouse Ostn cDNA was inserted into the EcoRI site of the pSG2 vector, which was produced by subcloning of the HindIII-XhoI fragment from pLG1-SAP containing the SAP promoter and rabbit $\beta$-globin gene (28) into pBluescript vector, resulting in the SAPOstn fusion gene. Next, the NotI-XhoI fragment of the fusion gene (SAP-Ostn transgene, $2.3 \mathrm{~kb}$ ) was purified and microinjected into the pronucleus of C57BL/6 mouse embryos using standard techniques. Among delivered mice, founders were identified by PCR genotyping with a pair of primers to amplify the transgene sequence (forward: TATTCTCCAAGTCCCTCTTTCCCC; reverse: AGAGAAGAGGGACAGCTATGACTG). They were mated with C57BL/6 mice, and we finally established 5 lines of SAP-Ostn-Tg mice, namely lines 3, 5, 33,44 , and 51 . The heterozygosity of the transgenic mice was maintained. In order to determine the transgene copy number in each line, genomic DNA was extracted from tail tips and purified with phenol/chloroform/isoamyl alcohol (Wako Pure Chemical). Quantitative real-time PCR was performed on an Applied Biosystems 7300 Real-Time PCR system with 1 pair of primers, designed based on exon 3 of the mouse Ostn gene to amplify both the Ostn coding region of genomic DNA and Ostn cDNA (forward: ACCCACAGCCAGAGAAGAGA; reverse: CCAGCTGAGAGTCTGTCAAGG). The estimated mouse Ostn gene level was normalized to Gapdh (forward: TGCACCACCAACTGCTTAGC; reverse: GGATGCAGGGATGATGTTCTG) for each sample. We evaluated the copy number of Ostn in transgenic mice by dividing the PCR values from transgenic mice by those from WT mice. Then the transgene copy number of each transgenic line was defined as the number that remained after 2 was subtracted from the number measured above. We used heterozygous SAP-Ostn-Tg mice for all of the experiments.

NPR-C-knockout $\left(\mathrm{Npr}^{---}\right)$mice were a gift from Nobuyuki Takahashi (Tohoku University, Sendai, Miyagi, Japan). Generation of CNP-knockout ( $N p p \mathrm{c}^{-/}$) mice (3) and SAP-CNP-transgenic (SAP$N p p c-T g$ ) mice (5) was reported previously.

To generate $\mathrm{Nppc}^{-/}$mice carrying the SAP-Ostn transgene (Nppc//SAP-Ostn-Tg mice), $N p p c^{+/-}$mice were mated with SAPOstn-Tg mice (line 51), and Nppc/-/SAP-Ostn-Tg mice were obtained. These mice were then mated with $N p p c^{+-}$mice. Among the resultant offspring, $\mathrm{Nppc}^{-/-}$mice and $\mathrm{N} p p \mathrm{c}^{-/} / \mathrm{SAP}-\mathrm{Ostn}-\mathrm{Tg}$ mice were identified by PCR genotyping, and both were used in this study.

To generate $\mathrm{Npr}^{--}$mice carrying the SAP-Ostn transgene (Npr3-/ ${ }^{-/ S A P-O s t n-T g ~ m i c e), ~} \mathrm{Npr}^{-/-}$mice were mated with SAPOstn-Tg mice (line 51), and $\mathrm{Npr}^{+/-} / \mathrm{SAP}$-Ostn-Tg mice were obtained. These mice were then mated with $\mathrm{Npr}^{-1-}$ mice. Among the resultant offspring, $\mathrm{Npr}^{-/-}$mice and $\mathrm{Npr} 3^{-/} / \mathrm{SAP}-\mathrm{Ostn}$ - Tg mice were identified by PCR genotyping and both were used in this study.

To generate mice carrying both $S A P-N p p c$ and SAP-Ostn transgenes (SAP-Nppc-Tg/SAP-Ostn-Tg mice), male SAP-Nppc-Tg mice were mated with female $S A P$-Ostn- $T g$ mice (line 51). Among the resultant offspring, double-transgenic SAP-Nppc-Tg/SAP-Ostn-Tg mice heterozygous for both SAP-Nppc and SAP-Ostn transgenes, SAP-Nppc-Tg mice, SAP-Ostn$\mathrm{Tg}$ mice, and WT mice were obtained and used for further experiments.

All animals were housed at $23^{\circ} \mathrm{C}$ in a $14: 10$-hour light/dark cycle with free access to water and standard chow.

Transgene expression. Total RNA was isolated from liver, cartilage, gastrocnemius muscle, brain, heart, lung, pancreas, intestine, kidney, and testis of 6-week-old mice using RNeasy Mini Kit (Qiagen). To prevent mixing with genomic DNA, cDNA was synthesized from total RNA using ReverTra Ace with genomic DNA Remover (Toyobo). PCR amplification of cDNA was performed on a GeneAmp PCR System 9700 (Applied Biosystems). To detect transgene expression, a forward primer was set in the untranslated sequence of PSG2 vector, and a reverse primer was set in the mouse Ostn cDNA sequence (forward: GCTGGTTGTTGTGCTGTCTC; reverse: CCAGCTGAGAGTCTGTCAAGG). Internal control RT-PCR was performed with Gapdh.

Quantitative real-time RT-PCR. Total RNA was isolated from epiphyseal cartilage of tibiae, lumbar vertebrae, and blood vessels of 3- and 6-week-old WT mice and from epiphyseal cartilage of tibiae and lumbar vertebrae of 3-week-old SAP-Ostn-Tg mice using RNeasy Mini Kit (Qiagen), and cDNA was synthesized using ReverTra Ace (Toyobo). Quantitative real-time RT-PCR was performed using SYBR Green dye on an Applied Biosystems 7300 Real Time PCR system. Data from epiphyseal cartilage of tibiae and lumbar vertebrae were normalized using hypoxanthine guanine phosphoribosyl transferase (Hprt) and peptidylprolyl isomerase A (Ppia) as reference genes, which were reported to be the most suitable genes for normalization in quantitative PCR assay in chondrocyte (29), and those from blood vessels were normalized to Gapdh and actin, beta $(A c t b)$ mRNA levels. The primers used in this analysis were as follows: $N p p c$, forward AGCGGTCTGG- 
GATGTTAGTG and reverse CCTCCССTCCCCAAATAATA; Npr3, forward TGACACCATTCGGAGAATCA and reverse TTTCACGGTCCTCAGTAGGG; Npr2, forward GAGGCCCTGCTGTACCAAATTCTA and reverse GAAGCCCACGATGTCACTGAAG; Hprt, forward GGACCTCTCGAAGTGTTGGATAC and reverse GCTCATCTTAGGCTTTGTATTTGGCT; Ppia, forward CGCGTCTCCTTCGAGCTGTTTG and reverse TGTAAAGTCACCACCCTGGCACAT; Gapdh, forward TGCACCACCAACTGCTTAGC and reverse GGATGCAGGGATGATGTTCTG; Actb, forward CATCCGTAAAGACCTCTATGCCAAC and reverse ATGGAGCCACCGATCCACA.

Growth curve. After mice reached 3 weeks of age, body weight and body length (naso-anal length and naso-tail length) were measured under anesthesia with isoflurane every week until they grew to 9 or 10 weeks of age.

Skeletal analysis. Mice were subjected to soft x-ray analysis (25 $\mathrm{kV}, 2.5 \mathrm{~mA}$ for 1 minute; Sofron type SRO-M50; Sofron), and then the anteroposterior and transverse diameters of the cranial bone, the length of the lumbar spine, and the lengths of the left humerus, radius, ulna, femur, and tibia of each mouse were measured on the soft x-ray film. We defined the sum of the lengths of L2, L3, L4, and L5 as the length of the lumbar spine.

Histological analysis. The left tibiae of mice were fixed in $10 \%$ formaldehyde neutral buffer solution (Nacalai Tesque). They were decalcified with $10 \%$ EDTA, embedded in paraffin, and sliced to expose the sagittal section for analyzing growth plates. Alcian blue and $\mathrm{H} \& \mathrm{E}$ staining and immunohistochemical staining for type X collagen of tibial growth plates with rat anti-type $\mathrm{X}$ collagen antibody (cata$\log$ LB-0092, lot 812021; LSL Co.) were performed, and sections were visualized under a Zeiss Axio Imager A1 microscope (Carl Zeiss). We subsequently measured the whole width and the width of the hypertrophic zone of tibial growth plates using the image analysis software AxioVision (version 4.6.3; Carl Zeiss).

Bone microstructural analysis. Microstructural analysis of the distal metaphyses of femurs was performed with micro-CT (SMX-100CTSV3; Shimadzu Co.) as previously described (30). The bone volume fraction, trabecular thickness, trabecular number, and trabecular separation were calculated using 3D trabecular bone analysis software (TRI/3D-BON; Ratoc System Engineering Co.).

Measurement of serum bone metabolic markers. Serum levels of osteocalcin (Alfa Aesar) and TRAP5b (Immunodiagnostic Systems) in mice were measured by ELISA using a commercial assay kit.

Measurement of plasma OSTN and CNP levels. Mouse blood samples were collected in microtubes containing $1 \mathrm{mg}$ EDTA-2Na (Thermo Fisher Scientific) and 0.6 trypsin inhibitor units aprotinin (Wako Pure Chemical) for $1 \mathrm{ml}$ of blood and stored on ice immediately. After centrifugation at $1,500 \mathrm{~g}$ for 20 minutes at $4^{\circ} \mathrm{C}$, plasma samples were collected and stored at $-80^{\circ} \mathrm{C}$ until use. Plasma OSTN concentrations were measured by sandwich chemiluminescence enzyme immunoassay (CLEIA), modifying the previously reported protocol for mouse ANP CLEIA (31). Anti-mouse OSTN C-terminus-directed antibody (anti-mMUS1G2) was raised in rabbits against immune complexes conjugated to a custom-synthesized C-terminal octadecapeptide (YGIPMDRIGRNRLSSSRG, $\mathrm{N}$-tyrosinyl mouse preproosteocrin [114 to 130 amino acids]) with the keyhole limpet hemocyanin. This antibody specifically recognizes the C-terminal sequence of mouse OSTN, showing only $0.2 \%$ affinity to human OSTN and no cross-reactivity with ANP, BNP, or CNP at the 1/450,000 dilu- tion. Anti-mouse/human OSTN rat mAb (catalog MAB2620, clone 311417), purchased from R\&D Systems, was prepared against mouse prepro-OSTN (28 to 130 amino acids) and recognizes the middle regions of both mouse and human OSTN. These 2 antibodies were used for the capture and detection antibody, respectively. The 96-well white plates (437796, Nunc C96 White MaxiSorp Immunoplate; Thermo Fisher Scientific) were coated with anti-mMUS1G2 $(0.5 \mu \mathrm{g} /$ well) in carbonate-bicarbonate buffer $(\mathrm{pH}$ 9.6) at room temperature overnight. The coated plates were washed with PBS containing $0.05 \%$ Tween-20 and blocked with $25 \%$ BlockAce (DS Pharma Biomedical) in PBS at room temperature for 30 minutes. Mouse OSTN peptide (80 to 130 amino acids) (Phoenix Pharmaceuticals) was used as a standard. Standards and plasma samples were applied to the coated plates and incubated at $4^{\circ} \mathrm{C}$ overnight. On the next day, after additional incubation at room temperature for 1 hour, plates were washed and incubated with MAB26260 at room temperature for 1 hour. Plates were washed and incubated with alkaline phosphatase-conjugated donkey anti-rat antibody (catalog 712-055153, lot 74624; Jackson ImmunoResearch Laboratories) at room temperature for 1 hour. For chemiluminescence measurements, plates were washed and incubated with substrates (CDP-Star Ready-ToUse with Emerald-II; Thermo Fisher Scientific) at room temperature for 30 minutes. The chemiluminescence signal intensity of each well at $535 \mathrm{~nm}$ was measured by 2030 ARVO-X3 (PerkinElmer). Samples were measured in duplicate, and the OSTN concentration was calculated from the standard curve obtained by regression analysis of 6 known concentration samples.

For the measurement of plasma CNP levels, CNP extraction was carried out using Sep-Pak C18 cartridges (Waters). After loading and washing, peptides were eluted with $50 \%$ acetonitrile (Nacalai Tesque) in $0.1 \%$ trifluoroacetic acid (Nacalai Tesque). Plasma CNP concentrations were measured using our newly developed sandwich CLEIA system against CNP-53, which has a detection limit down to $0.25 \mathrm{pM}$ and no cross-reactivity with ANP, BNP, and other related peptides (Minamino et al., unpublished observations).

Measurement of cGMP levels. cGMP levels in lumbar vertebrae of 3-week-old WT, SAP-Nppc-Tg, and SAP-Ostn-Tg mice were measured by cGMP ELISA kit (Cayman Chemical). Tissues were homogenized in 5\% trichloroacetic acid, and cyclic nucleotides were extracted using water-saturated ether. Samples were acetylated to increase the sensitivity of the assay before they were used.

Statistics. All results are expressed as the mean \pm SEM. Statistical analysis was performed using either 2-tailed Student's $t$ test or 1-way ANOVA with the Tukey-Kramer test. Statistical significance was defined as $P<0.05$.

Study approval. Maintenance of the mice and all experimental procedures were performed in accordance with the ethical guidelines of Kyoto University and the United States NIH Guide for the Care and Use of Laboratory Animals (National Academies Press, 2011). This study was approved by the Animal Research Committee, Graduate School of Medicine, Kyoto University.

\section{Author contributions}

YK generated and analyzed the data. YK and AY designed the experiments and wrote the manuscript. KPM and HY measured plasma OSTN concentrations of mice. CNO and N. Minamino measured plasma CNP concentrations of mice. SY and KN pro- 
vided CNP-knockout mice. KPM, HWT, YY, KH, YU, IY, EK, YS, TF, HY, MM, N. Mochizuki, and NI contributed to the discussion and reviewed the manuscript.

\section{Acknowledgments}

We thank Nobuyuki Takahashi for providing the NPR-C-knockout mice. This work was supported by a Japan Society for the Promotion of Science KAKENHI grant (26461381 to AY) and by Japan Agency for Medical Research and Development Japan Agency for Medical Research and Development-Core Research for Evolutionary Medical Science and Technology (AMED-CREST) grants (15652238 to HY, 13414779 to N Mochizuki).
Address correspondence to: Akihiro Yasoda, Department of Diabetes, Endocrinology and Nutrition, Kyoto University Graduate School of Medicine, 54 Shogoin, Kawahara-cho, Sakyo-ku, Kyoto 606-8507, Japan. Phone: 81.75.751.3181; E-mail: ayasoda@kuhp. kyoto-u.ac.jp.

C. Nagai-Okatani's present affiliation is: Biotechnology Research Institute for Drug Discovery, National Institute of Advanced Industrial Science and Technology, Tsukuba, Ibaraki, Japan.

N. Minamino's present affiliation is: Omics Research Center, National Cerebral and Cardiovascular Center, Osaka, Japan.
1. Berendsen AD, Olsen BR. Bone development. Bone. 2015;80:14-18.

2. Kronenberg HM. Developmental regulation of the growth plate. Nature. 2003;423(6937):332-336.

3. Chusho H, et al. Dwarfism and early death in mice lacking C-type natriuretic peptide. Proc Natl Acad Sci U S A. 2001;98(7):4016-4021.

4. Yasoda A, et al. Overexpression of CNP in chondrocytes rescues achondroplasia through a MAPK-dependent pathway. Nat Med. 2004;10(1):80-86.

5. Kake T, et al. Chronically elevated plasma C-type natriuretic peptide level stimulates skeletal growth in transgenic mice. Am JPhysiol Endocrinol Metab. 2009;297(6):E1339-E1348.

6. Nakao K, Ogawa Y, Suga S, Imura H. Molecular biology and biochemistry of the natriuretic peptide system. II: Natriuretic peptide receptors. J Hypertens. 1992;10(10):1111-1114.

7. Tamura N, Doolittle LK, Hammer RE, Shelton JM, Richardson JA, Garbers DL. Critical roles of the guanylyl cyclase B receptor in endochondral ossification and development of female reproductive organs. Proc Natl Acad Sci U S A. 2004;101(49):17300-17305.

8. Tsuji T, Kunieda T. A loss-of-function mutation in natriuretic peptide receptor $2(\mathrm{Npr} 2)$ gene is responsible for disproportionate dwarfism in $\mathrm{cn} / \mathrm{cn}$ mouse. J Biol Chem. 2005;280(14):14288-14292.

9. Bartels CF, et al. Mutations in the transmembrane natriuretic peptide receptor NPR-B impair skeletal growth and cause acromesomelic dysplasia, type Maroteaux. Am J Hum Genet. 2004;75(1):27-34.

10. Hachiya $\mathrm{R}$, et al. Intact kinase homology domain of natriuretic peptide receptor-B is essential for skeletal development. JClin Endocrinol Metab. 2007;92(10):4009-4014.

11. Olney RC, et al. Heterozygous mutations in natriuretic peptide receptor-B (NPR2) are associ- ated with short stature. JClin Endocrinol Metab. 2006;91(4):1229-1232.

12. Vasques GA, et al. Heterozygous mutations in natriuretic peptide receptor-B (NPR2) gene as a cause of short stature in patients initially classified as idiopathic short stature. J Clin Endocrinol Metab. 2013;98(10):E1636-E1644.

13. Amano N, et al. Identification and functional characterization of two novel NPR2 mutations in Japanese patients with short stature. J Clin Endocrinol Metab. 2014;99(4):E713-E718.

14. Wang SR, et al. Heterozygous mutations in natriuretic peptide receptor-B (NPR2) gene as a cause of short stature. Hum Mutat. 2015;36(4):474-481.

15. Hisado-Oliva A, et al. Heterozygous NPR2 mutations cause disproportionate short stature, similar to Léri-Weill Dyschondrosteosis. JClin Endocrinol Metab. 2015;100(8):E1133-E1142.

16. Hisado-Oliva A, et al. Mutations in C-natriuretic peptide (NPPC): a novel cause of autosomal dominant short stature [published online ahead of print June 29, 2017]. Genet Med. https://doi. org/10.1038/gim.2017.66.

17. Miura K, et al. An overgrowth disorder associated with excessive production of cGMP due to a gainof-function mutation of the natriuretic peptide receptor 2 gene. PLoS One. 2012;7(8):e42180.

18. Miura K, et al. Overgrowth syndrome associated with a gain-of-function mutation of the natriuretic peptide receptor 2 (NPR2) gene. Am JMed Genet A. 2014;164A(1):156-163.

19. Bonafe L, et al. Nosology and classification of genetic skeletal disorders: 2015 revision. Am J Med Genet A. 2015;167A(12):2869-2892.

20. Thomas G, et al. Osteocrin, a novel bone-specific secreted protein that modulates the osteoblast phenotype. J Biol Chem. 2003;278(50):50563-50571.

21. Nishizawa $\mathrm{H}$, et al. Musclin, a novel skeletal muscle-derived secretory factor. J Biol Chem.
2004;279(19):19391-19395.

22. Moffatt $\mathrm{P}$, et al. Osteocrin is a specific ligand of the natriuretic peptide clearance receptor that modulates bone growth. J Biol Chem. 2007;282(50):36454-36462.

23. Kita S, et al. Competitive binding of musclin to natriuretic peptide receptor 3 with atrial natriuretic peptide. J Endocrinol. 2009;201(2):287-295.

24. Nakao K, et al. The Local CNP/GC-B system in growth plate is responsible for physiological endochondral bone growth. Sci Rep. 2015;5:10554.

25. Ogawa Y, et al. Molecular cloning of the complementary DNA and gene that encode mouse brain natriuretic peptide and generation of transgenic mice that overexpress the brain natriuretic peptide gene. J Clin Invest. 1994;93(5):1911-1921.

26. Nakao K, et al. Endothelium-derived C-type natriuretic peptide contributes to blood pressure regulation by maintaining endothelial integrity. Hypertension. 2017;69(2):286-296.

27. Matsukawa N, et al. The natriuretic peptide clearance receptor locally modulates the physiological effects of the natriuretic peptide system. Proc Natl Acad Sci U S A. 1999;96(13):7403-7408.

28. Toyonaga $\mathrm{T}$, et al. Chronic active hepatitis in transgenic mice expressing interferon- $\gamma$ in the liver. Proc Natl Acad Sci U S A. 1994;91(2):614-618.

29. Zhai Z, Yao Y, Wang Y. Importance of suitable reference gene selection for quantitative RT-PCR during ATDC5 cells chondrocyte differentiation. PLoS One. 2013;8(5):e64786.

30. Ueda Y, et al. C-type natriuretic peptide restores impaired skeletal growth in a murine model of glucocorticoid-induced growth retardation. Bone. 2016;92:157-167.

31. Nagai C, Minamino N. Direct chemiluminescent enzyme immunoassay for atrial natriuretic peptide in mammalian plasma using a PEGylated antibody. Anal Biochem. 2014;461:10-16. 